\title{
Convergencias disciplinares entre historia $y$ antropología: la perspectiva "histórico-regional" y las investigaciones sobre los pueblos indígenas en Norpatagonia, Argentina
}

\author{
Sebastián Valverde, Universidad de Buenos Aires, Argentina
}

\begin{abstract}
Resumen: El presente artículo analiza el proceso de convergencia disciplinar entre la Historia y la Antropología desarrollado en el ámbito académico argentino, a partir de las investigaciones sobre los pueblos indígenas en la zona Norte de la Patagonia en Argentina. Con este objetivo se indagan en los aportes teóricos y metodológicos efectuados desde la perspectiva "histórico-regional" como forma de interpretar procesualmente las dinámicas de diversa índole que surgen a partir de la labor etnográfica, característica de la Antropología Social. El trabajo expone, en primer lugar, una contextualización acerca del proceso interdisciplinar analizado, teniendo en cuenta sus aspectos más generales y los antecedentes en nuestro pais, con énfasis en la producción de diferentes equipos de investigadores -su surgimiento y consolidación- a nivel nacional. En segundo lugar, se considera la recuperación de diversas categorías y lecturas de los "enfoques histórico-regionales" para el abordaje de los movimientos indígenas. En particular, se retoma esta problemática en función del trabajo de investigación que desde fines de los años noventa venimos realizando en torno a la emergencia, desarrollo y consolidación del pueblo Mapuche en Argentina.
\end{abstract}

Palabras clave: antropología, historia, enfoque histórico-regional, interdisciplinariedad, Patagonia argentina, pueblo Mapuche

\begin{abstract}
This article analyzes the disciplinary convergence process between history and anthropology developed in the Argentine academic context, based on the research on indigenous peoples on the North of the Argentine Patagonia. For such purposes, we look into the theoretical and methodological contributions made by the "historical-regional" perspective as a way of interpreting the process of the different dynamics that arise from the ethnographic work performed by social anthropology. The article deals, in the first place, with a contextualization of the interdisciplinary process analyzed, taking into account its most general aspects and the antecedents in our country, emphasizing the production of different research teams, its creation and consolidation, at a national level. Secondly, we explore the recovery of different categories and "historical-regional approach" literature in order to deal with indigenous movements. In particular, we approach this issue based on the research work that we have been carrying out since the 90 s on emergency, development and consolidation of the Mapuche people in Argentina
\end{abstract}

Keywords: Anthropology, History, Historical-Regional Approach, Interdisciplinarity, Patagonia Argentina, Mapuche People

\section{Presentación}

"Es cierto que los seres humanos crean estructuras sociales y que conceden significado a los acontecimientos; pero estas estructuras y significados poseen orígenes históricos que conforman, delimitan y ayudan a explicar esa creatividad".

(Sidney Mintz, Dulzura y poder. El lugar del azúcar en el mundo moderno, 1996: 28)

E n el año 1899 el historiador de derecho inglés Frederic William Maitlan -en una conferencia destinada a volverse famosa- expresó que la Antropología se encontrará frente a la elección "entre ser Historia o no ser nada" (Viazzo; 2003: 17). En los siguientes cincuenta años, quedó claro que la Antropología eligió resueltamente no ser Historia, sin por ello devenir "en nada". 
Durante una parte importante del Siglo XX, la Antropología y la Historia transcurrieron por caminos separados, ya que la primera era "ahistórica", en tanto la segunda tendía a ser "acultural". Esta separación entre ambas disciplinas se dio especialmente entre las décadas de 1920 y 1950, época de hegemonía de la escuela funcionalista británica, que evidenció un profundo desinterés hacia el análisis diacrónico. Hacia la mitad del Siglo XX esta tendencia comienza a revertirse, manifestándose una creciente insatisfacción con los abordajes sincrónicos. Prueba de ello, es que desde la propia escuela estructural-funcionalista británica, Edward Evans Pritchard empieza a introducir el componente histórico en sus análisis. En consecuencia, diversos factores diferenciados -y a la vez interrelacionados- convergen, al mismo tiempo que se debilitan las "fronteras" entre ambas disciplinas.

Por un lado, los Historiadores empiezan a advertir la necesidad de "ver a la Antropología", como lo señalara Keith Thomas en el año 1963 -invirtiendo la polémica aseveración de Maitland medio Siglo atrás- al afirmar “(...) que la Historia se encontraba ahora en el deber de escoger entre ser Antropología o no ser nada" (Viazzo, 2003: 18). Desde ya, tampoco la Historia se transformó en Antropología, pero muchos historiadores comenzaron a retomar sus métodos y a abordar otros grupos sociales y problemas más cercanos a los tradicionalmente analizados por la Antropología. Así es como en las últimas décadas se fueron desarrollando novedosos campos de la historiografía que mantienen mayores puntos de contacto con la forma antropológica de abordar el mundo social. Es el caso de la Historia social, la Historia oral, los estudios subalternos, la Historia de las mentalidades y la Historia cultural -entre otras- (Rockwell: 2009). Es necesario destacar, en este sentido, el aporte de la "Escuela de los Annales" desde los años treinta, con destacados investigadores como Marc Bloch, Lucien Febvre y Fernand Braudel.

Por otro lado, el creciente conocimiento de las limitaciones y distorsiones causadas por las orientaciones metodológicas sincrónicas o atemporales, ha llevado a un "retorno a la Historia", afectando este cambio a todas las ciencias humanas (Viazzo, 2003: 18). La Antropología -que nació planteando una fuerte ruptura epistemológica con el estudio temporal de los fenómenos sociales- como es de esperar, no fue ajena a esta dinámica. Así es como se fue modificado el concepto estático y sistémico de cultura que dominaba la disciplina, para descubrir cómo las configuraciones culturales aparentemente inmutables han sido producidas, reproducidas y transformadas bajo condiciones históricas particulares (Rockwell, 2009). En este sentido, cabe destacar los aportes de autores de corrientes muy disímiles entre sí, que fueron trabajando en esta línea, como Evans Pritchard, Bonfil Batalla, Sahlins, Roseberry y Wolf (Rockwell, 2009).

En Argentina, esta es la tendencia que se viene experimentando desde el devenir democrático de los años ochenta y la consolidación de múltiples centros de investigación en diferentes ciudades del país. En los últimos años, esto ha tenido un impulso con la ampliación en la cantidad de investigadores -fundamentalmente a través de un número mayor de becas-, publicaciones y una mayor oferta en el dictado de carreras sociales y humanísticas, además de la apertura de nuevas universidades.

En la región cordillerana del Norte de Patagonia $^{1}$ en que desarrollamos nuestras investigaciones (ver croquis 1), viene registrándose en los últimos años una loable convergencia entre la Historia y otras disciplinas sociales -una de ellas nuestro campo profesional, la Antropología-. Esto es explicable, ya que en la ciudad de Neuquén (capital de la

\footnotetext{
${ }^{1}$ La Patagonia constituye la parte más austral de América del Sur, que comprende territorios del sur de Chile y de Argentina. Al este de la Cordillera de los Andes - que oficia de divisoria entre los dos países- se encuentra la parte argentina de la Patagonia y al oeste la fracción chilena. En nuestro país, Argentina, la Patagonia abarca un sector de la provincia de Buenos Aires, junto con las provincias de La Pampa, Neuquén, Río Negro, Chubut, Santa Cruz y Tierra del Fuego (ver Croquis II). Las cuatro primeras provincias corresponden al sector norte de la Patagonia, que abordamos en nuestras investigaciones y al que nos referimos en este trabajo (específicamente la región cordillerana, ver croquis I). En cambio el sector sur está compuesto por las últimas tres provincias antes destacadas. En total representa un territorio de aproximadamente $800.000 \mathrm{~km}^{2}$, es decir, algo menos de un tercio de los $2.780 .400 \mathrm{~km}^{2}$ de la superficie argentina.
} 
Provincia homónima) desde hace varios años se dicta la carrera de Historia y hay diversos grupos consolidados de investigadores, situación que difiere de otras carreras afines (Sociología, Antropología, etc.), cuyo dictado en la zona es mucho más reciente, y el desarrollo de equipos profesionales es incipiente. Esta situación permite entender la influencia de la disciplina histórica sobre las restantes.

En el presente artículo, que surge en función de los propósitos del Séptimo Congreso Internacional sobre Ciencias Sociales Interdisciplinares, nos proponemos indagar sobre el proceso de convergencia entre la Historia y la Antropología, a partir de los estudios que abarcan la zona Norte de Patagonia. Consideramos, para ello, los aportes en términos teóricos y metodológicos que viene efectuando el denominado enfoque "histórico-regional" a la Antropología.

Abordaremos esta problemática, a partir del trabajo de investigación que desde fines de los años noventa venimos realizando en dicha zona, referido a múltiples aspectos del pueblo indígena Mapuche ${ }^{2}$. Entre ellos se encuentran los efectos de diversas actividades socioeconómicas -como el turismo- los conflictos territoriales, la génesis y desarrollo de las organizaciones indígenas. También desde hace varios años venimos trabajando -en forma conjunta con integrantes de dichas comunidades y organizaciones indígenas- en diversos proyectos de intervención, centrados en la recuperación de testimonios orales de los pobladores sobre sus vivencias históricas.

El recorrido que proponemos plantea un ida y vuelta entre los estudios de caso, y la reflexión teórica que fuimos problematizando acerca de esta convergencia interdisciplinar. Efectuamos este análisis para el propio camino investigativo, y para otros trabajos que vienen retomando esta mirada en la región.

\section{Antecedentes de los estudios históricos-regionales en Argentina}

Para comprender el desarrollo de los denominados "estudios históricos regionales" -y su influencia en las investigaciones efectuadas en la región de Norpatagonia desde diferentes disciplinas, tales como la Antropología, la Arqueología, la Geografía, la Sociología, etc.- es necesario remitirse a la profunda revisión historiográfica por la que ha atravesado la Historia Argentina, producto de la aguda crisis de los paradigmas imperantes en décadas pasadas. En nuestro país fue, a partir del regreso de la democracia en el año 1983, cuando irrumpen las Historias regionales a manera de "Historias compensatorias" (Méndez y Tozzini, 2011).

Es en este contexto donde dicho abordaje ha alcanzado una dimensión significativa en los últimos años. Aquí es donde se sitúa el esfuerzo por reconstruir los procesos históricos de las diversas regiones del interior del país, trabajo indudablemente ligado al fortalecimiento de algunos centros académicos con fuerte contenido regional. Esto no quiere decir que no haya habido anteriormente producción historiográfica vinculada a la "Historia regional", pero, en general se entendían por ello los tratamientos acotados a las "Historias provinciales" -de índole institucional- donde no se manifestaba un particular interés por definir otros espacios de análisis históricos más amplios (Bandieri, 2001a).

La definición "apriorística" del objeto de estudio -que precisamente desecha el enfoque regional- es la muestra más elocuente de la influencia de la geografía neopositivista y su definición de región como un objeto de estudio en sí mismo. Así, la Historia de la "región" no era otra cosa que la sumatoria de los límites políticos provinciales supuestamente involucrados en ella (Bandieri, 2001a). En la crítica efectuada por Carbonari (2009: 19) a dichas lecturas:

\footnotetext{
2 "Mapu" significa en "mapudungún", (en su lengua originaria) "tierra" y "che" significa "gente", así "Mapuche" quiere decir "Gente de la tierra" (Juliano, 1996). Este pueblo indígena que se asienta en el sur de Chile y de Argentina, sobrevivió a los ataques genocidas y etnocidas llevados a cabo a ambos lados de la cordillera de los Andes a fines del Siglo XIX (Radovich y Balazote, 2009). En el vecino país, Chile, se asientan en la Octava, Novena y Décima Región y (como resultado de las migraciones) en la región Metropolitana, sumando un millón de integrantes (Juliano, 1996), siendo en este caso el grupo indígena más significativo del país. En Argentina, se asientan en las provincias de Chubut, Río Negro, Neuquén, La Pampa y Buenos Aires (Radovich, 2003) conformando algo más de 100.000 miembros, lo que hace que sea uno de los pueblos originarios más importantes (INDEC, 2004-2005).
} 
“(...) se pretendía hacer coincidir la Historia con la territorialidad de la jurisdicción estatal”. Remarcamos el carácter funcionalista de este tipo de explicación, donde las partes constituyen el todo y este último es visualizado exclusivamente como la sumatoria de las primeras. Desde ya, lo mismo sucedía con la "Historia nacional" -concebida como una adición de las diferentes regiones que la integraban- con una lectura sesgada hacia las regiones dominantes (Favaro y Scuri, 2005: 19).

A partir de las limitaciones de tales perspectivas, y con el fin de alcanzar niveles explicativos más adecuados, es que diversos historiadores comenzaron a preguntarse sobre la forma de construir una Historia regional en términos más comprensivos, que pudiera poner en suspenso alguno de los preceptos más recurrentes y no contrastados de la Historia nacional.

En estos planteamientos innovadores tuvieron una gran influencia las formulaciones del estructuralismo francés, de la denominada Escuela de los Annales, donde “(...) los estudios regionales dejaban de ser datos en sí mismos de la realidad para implicar conceptos relacionales que se explicaban en función de un contexto mayor" (Carbonari, 2009: 23). A partir de estos preceptos, se produjo: "(...) un desplazamiento del empirismo por la teoría" (Carbonari, 2009: 23). Un antecedente ineludible lo constituyen las obras de Braudel del año 1949, El Mediterráneo y el mundo del Mediterráneo en la época de Felipe II, trabajo que se transformó en referente para los estudios regionales europeos del grupo de Annales. Esta obra, además, adquiere una importancia decisiva por el entendimiento del espacio en su relación con el tiempo, inaugurando un nuevo diálogo entre la Historia y la Geografía. Continuando con la caracterización efectuada por Carbonari (2009), otro antecedente es el libro de Chevalier del año 1952 La formación de los latifundios en México: Tierra y sociedad en los siglos XVI y $X V I I$. Estos dos autores se adscriben a una aproximación funcional-estructuralista. Pero, también se destaca entre estos antecedentes, la obra de Maurice Dobb (del año 1946), Estudio sobre el desarrollo del capitalismo, en este caso referenciándose con el enfoque marxistaestructuralista (Carbonari, 2009). En ese contexto de debate, fue significativa para América Latina la compilación (en la década del setenta) de Assadourian (1982), Modo de producción en América Latina, que reunió artículos de distintos autores que participaban de discusiones de la época desde estas perspectivas (Ernesto Laclau, Ciro Cardoso, Juan Carlos Garavaglia, entre otros). Posteriormente, Assadourian (1982) estudió la producción cordobesa entre los siglos XVI y XVII y los vínculos que establecía con lo que denominó el "espacio peruano". El trabajo de Assadourian posteriormente se transformaría en un modelo referencial para los estudios regionales del mundo colonial (Carbonari, 2009). Otros autores a destacar son Cardoso (1984), Garavaglia (1983), Chiaramonte (1983) y Donghi (1972) -este último se ha abocado a la Historia argentina. (Carbonari, 2009)

En nuestro país, ha contribuido a consolidar esta línea, la traducción y publicación del trabajo de Eric Van Young: Haciendo Historia regional. Consideraciones metodológicas y teóricas ${ }^{3}$. Uno de los aportes más destacados de este autor, ha sido el hecho de considerar a la región “(...) como la ‘espacialización’ de una relación económica” (Van Young, 1991: 257) en razón de lo cual “(...) debía otorgarse especial atención a las relaciones de mercado vigentes en cada momento histórico" (Bandieri, 2001a: 5).

Esta interpretación tuvo amplia difusión y aceptación en Argentina, entre quienes intentaban aproximarse a los diversos estudios regionales. Sostiene Van Young que una definición funcional muy simple del concepto de región: “(...) sería la de un espacio geográfico con una frontera que lo activa, la cual estaría determinada por el alcance efectivo de algún sistema cuyas partes interactúan más entre sí que con los sistemas externos" (1991: 257-258).

Otro autor -del cual Bandieri remarca la gran influencia que ha tenido en el abordaje histórico-regional local-, es el peruano Carlos Sempat Assadourian (que citamos anteriormen-

\footnotetext{
${ }^{3}$ El trabajo fue publicado en el Anuario del Instituto de Estudios Histórico Sociales $N^{\mathrm{o}}$ 2, de la Universidad del Centro de la Provincia de Buenos Aires, en el año 1987.
} 
te), en especial a partir del libro Mercado interno, regiones y espacio económico (1982). Desde este planteamiento, los espacios económicos debían reconstruirse en la investigación histórica atendiendo a un sistema de relaciones internas y externas que se modifican en cada período, uno de cuyos elementos sobresalientes era la circulación de mercancías, pero también el estudio de las relaciones políticas, económicas y sociales. Así, Assadourian descubría un vasto espacio socioeconómico integrado por diversos territorios que más tarde terminarían convirtiéndose en los respectivos Estados nacionales (Ecuador, Perú, Bolivia, Chile, Argentina y Paraguay), donde “(...) los factores historiográficamente analizados como 'externos', se transformaban comprensivamente en elementos 'internos' de la región misma” (Bandieri, 2001a: 5).

\section{El concepto de región}

En este apartado, retomaremos el concepto de región desde los enfoques histórico-regionales, que partiendo de una aguda crítica a las concepciones positivistas lo definieron como una unidad en sí misma. En palabras de de Jong:

Toda fragmentación territorial y temporal a priori es una aberración epistemológica, ya que solo el conocimiento del fenómeno como totalidad, conduce a entender el rol dinámico de las transformaciones geográficas a partir de entender el carácter organizador del comportamiento social. (2009: 42)

En efecto, desde la geografía tradicional: “(...) el espacio quedó recortado en regiones univocas definitivas, con límites -naturales o institucionales- absolutamente rígidos y con características propias pasibles de observación directa" (Bandieri, 1995: 279). Por ello, en contraposición con tales lecturas, sostiene esta autora:

(...) los límites jurídicos y/o geográficos resultan siempre inadecuados para la comprensión de los procesos socioeconómicos que se vuelven ininteligibles en la rigidez de un espacio dado ya que nunca empiezan y terminan en él. Las Historias locales, encerradas en límites provinciales o territoriales preestablecidos, no parecen entonces adecuadas si no es a partir de su inserción en ámbitos más comprensivos. (1995: 278-279)

En contraposición con la concepción tradicional, esta misma autora define (retomando a de Jong, 1991) que:

La región no es un 'dato' impuesto desde afuera, (...) la región se construye sobre la base de la dinámica relación hombre-espacio, que es una construcción social; obtenemos por resultado la necesidad de considerar entonces a la región como un sistema abierto, como un objeto que se aborda mediante sucesivas aproximaciones que apuntan en su conjunto a la idea de totalidad, donde los actores sociales cobran un rol protagónico en relación a los procesos de generación, apropiación y distribución del excedente económico. (Bandieri, 1995: 282)

Aquí, deseamos detenernos en varios aspectos de esta concepción de "región". En primer lugar, en el carácter interdisciplinario de esta recuperación conceptual efectuada desde estos abordajes alternativos. Desde el momento en que se delimita el espacio y el tiempo a estudiar, se involucra a la Geografía y a la Historia como campos disciplinarios. Máxime, tomando en cuenta que desde estas lecturas críticas al positivismo, se enfatiza que dichas unidades espaciotemporales son una "construcción social", y por lo tanto, su carácter es contingente. Como ha señalado de Jong “(...) ambas disciplinas [Geografía e Historia] se encontrarían frente al mismo desafío: construir espacialidades y periodizaciones no sólo flexibles, sino apropiadas para los problemas que se desean conocer" (de Jong, 1981, en Méndez y Tozzini, 2011).

En segundo lugar, otra implicación de este concepto es su variabilidad espacio-temporal. En efecto la región, se constituye "(...) a partir de las interacciones sociales que la definen 
como tal en el espacio y en el tiempo, dejando de lado cualquier delimitación previa que pretenda concebirla como una totalidad preexistente" (Bandieri, 2001a: 6).

El tercer aspecto inherente a esta definición es su no definición "a priori", tal como lo ha sintetizado Van Young, al destacar que “(...) las regiones son hipótesis a demostrar y que, cuando escribimos Historia regional, estaríamos tratando de hacer justamente eso, antes que describir entidades antecedentes" (1991: 257). En términos comparables lo plantean Favaro y Scuri, quienes parten del supuesto de no abordar a "(...) la región en términos conceptuales para estudiar ciertos procesos sino que al estudiar los procesos queda aclarado el espacio investigable" (2005: 11).

\section{Los abordajes histórico-regionales en Norpatagonia y la revisión de viejos preceptos}

En la región de Norpatagonia, es necesario destacar el proceso de surgimiento y consolidación de diferentes equipos de investigadores -principalmente de la disciplina histórica- del ámbito de la Universidad Nacional de Comahue, en especial en la ciudad de Neuquén, que luego se fueron estableciendo y ampliando a otras localidades ${ }^{4}$. En este sentido, cabe destacar los aportes de Bandieri (1993a y 1993b; 1995; 2001a; 2005 y 2009), Favaro y Scuri (2005), Méndez (2005, 2006, 2009 y 2010) y Navarro Floria (1999 y 2008). También cabe mencionar múltiples trabajos colectivos (Bandieri, et. al. 1993; et al. 2001b y et al. 2006 y Favaro, 2005). La publicación, en especial en la última década, de estos trabajos colectivos, evidencia el desarrollo y consolidación de múltiples equipos de profesionales, la incorporación de nuevos autores y generaciones de investigadores a estas perspectivas.

Estos aportes, se ven plasmados a través de diversas publicaciones como el Boletín del Departamento de Historia entre los años 1980 y 1990, que luego derivó en la Revista de Historia de la Facultad de Humanidades de la Universidad Nacional del Comahue (que lleva hasta el momento doce números editados). La creciente relevancia de estos debates convirtió "al Comahue" en una voz autorizada en todo lo relacionado con el enfoque regional y propició la consiguiente "nacionalización" de lo producido en este centro académico (Colectivo Editor Revista de Historia, 2011).

Otro caso paradigmático, ha sido la aparición de la revista Pueblos y Fronteras de la Patagonia Andina -editada en la localidad de El Bolsón, Provincia de Río Negro- de un importante nivel de difusión en la región. Se publicaron seis números entre los años 2001 y 2005 con trabajos de especialistas de diferentes disciplinas, recuperando estos marcos explicativos.

En relación a los eventos académicos, se realizaron las Jornadas de Historia de la Patagonia efectuadas en diferentes localidades de la región, los Congresos de Historia de la Patagonia Argentino-Chilena en la localidad Trevelín (Provincia de Chubut) y los Encuentros Regionales de Historia Oral. La creciente participación, periodicidad y ampliación de estos encuentros puso de manifiesto la consolidación de estas perspectivas alternativas en la región, el acercamiento de las nuevas generaciones de investigadores a las mismas y la repercusión en las disciplinas afines. Por último, cabe señalar los dos Talleres binacionales Argentino-Chileno Araucania-Norpatagonia: cultura y espacio efectuados en los años 2010 y 2011 -desde una mirada interdisciplinaria-, en la recientemente creada Universidad Nacional de Río Negro con sede en la ciudad de San Carlos de Bariloche.

\footnotetext{
${ }^{4}$ Es el caso de la ciudad de San Carlos de Bariloche -principal localidad cordillerana de Norpatagonia- donde desde hace unos años también se dicta dicha carrera y se vienen consolidando grupos de investigadores con crecientes producciones (Ver Méndez, 2005, 2006, y 2010), en especial (dada la localización geográfica de esta ciudad) referidas a la problemática de la dinámica de la frontera con Chile.
} 
Una serie de preceptos de los denominados enfoques "históricos regionales" han permitido comprender las múltiples dinámicas de la región de Norpatagonia y en ese proceso fueron derribando verdaderas "fronteras" instaladas por la ideología positivista en base a la cual se conformó el Estado-nación. En primer lugar, esta noción que señalábamos de la región como un sistema "abierto" -que no posee correspondencia con los límites jurídicos formalesadquiere particular relevancia en el contexto específico de Patagonia, ya que las provincias actuales surgieron de la división administrativa de los territorios nacionales del año 1885. Ellos fueron fijados, por aquel entonces, a partir de accidentes geográficos y trazos (como paralelos y meridianos) que no responden a criterio alguno de funcionamiento económico y cultural de las sociedades involucradas (Bandieri, 2005). Por ello, “(....) poco sirven a la hora de explicar el funcionamiento de lo social y de la infinidad de relaciones que lo superan" (Bandieri, 2005: $13)$.

El segundo aspecto que resulta central a la hora de comprender las dinámicas regionales (en especial en la zona cordillerana), es la estrecha vinculación entre las poblaciones asentadas en ambos países (Chile y Argentina), lo que implica -al decir de Mandrini- "(...) salir de los límites impuestos por una historiografía de tipo nacional o, más correctamente, nacionalista que, nacida al calor de los procesos constitutivos del Estado nación en la segunda mitad del siglo XIX, encuadró en los límites de esa "nación" -físicos, políticos y culturales- toda la Historia anterior" (2007: 25).

Esto incluye, analizar la naturaleza y dinámica de tales interrelaciones (económicas, familiares, sociopolíticas, etc.) que se daban entre los pueblos asentados a ambos lados de la cordillera, así como las relaciones que mantuvieron varias décadas después de la delimitación en muchos casos más formal que efectiva- de dichas jurisdicciones nacionales. Por ello, otro de los aportes centrales de estos trabajos, es que: "La cordillera dejó de ser el muro o la barrera que separaba esos mundos para convertirse en un espacio que los articulaba. [Sin perder de vista las especificidades] (...) comenzamos a pensar en una historia común, de pueblos indisolublemente unidos" (Mandrini, 2007: 25). Estos aportes, vienen demostrando a través de profundos estudios efectuados recientemente, cómo hasta bien entrado el Siglo XX existieron en la región áreas que lograron mantener cierta autonomía económica a través de la interrelación comercial con localidades del lado chileno (Bandieri, 2005; Méndez, 2005; Mandrini, 2007).

Un último aspecto que deseamos retomar del abordaje histórico-regional, es que también recupera la Historia indígena, que recién en las últimas décadas del Siglo XX se convirtió en materia de preocupación de los historiadores. El peso de la historiografía tradicional del Siglo XIX hizo que la Historia de estos pueblos apareciera casi siempre como (...) un capítulo introductorio y desvinculado del conjunto general (Bandieri, 2005: 14), asignándoles -por lo tanto- un rol marginal, y contribuyendo así la propia historiografía a su desaparición. Como acertadamente ha señalado Mandrini: "Si en el mito de la 'Argentina europea' esta era un país 'sin Indios', la Historia de esa Argentina debía también serlo o, en todo caso, los "nativos" eran sólo cosas del pasado, reliquias arqueológicas cuyo lugar estaba en los museos de Historia Natural creados en esos tiempos" (2007: 21). Por ello, también se les relegaba como objeto de estudio en forma exclusiva a antropólogos y arqueólogos, donde la frontera entre "blancos" e "indígenas", también se convertía en una frontera entre estas disciplinas y la Historia. En cambio, desde estas visiones alternativas, la Historia blanca e indígena transcurren paralelas, formando parte simultanea de la complejidad de los procesos histórico-sociales regionales (Bandieri, 2005; Mandrini, 2007). Pero además, desde las lecturas más recientes, lo que sucede en el mundo hispano criollo posee una importancia trascendental para el mundo indígena, dadas las vinculaciones y la interdependencia entre ambas sociedades. Basta con señalar " (...) el impacto que tuvo el triunfo del proyecto liberal en la década de 1860, que acabó con la destrucción de ese mundo [el indígena] y su anexión definitiva al Estado-nación" (Mandrini, 2007: 26). 


\section{Profundizando en los abordajes histórico-regionales y su repercusión en las restantes disciplinas}

En este apartado, nos proponemos ahondar en algunos lineamientos de los denominados "enfoques-regionales", que hacen más comprensible su repercusión y adopción de alguno de sus aspectos desde otras disciplinas.

Una de las características del método regional, es que se cimienta en un cariz inherentemente interdisciplinario (Méndez y Tozzini, 2011). De hecho resultaría un contrasentido pensar en la no fragmentación a priori de un determinado espacio geográfico o período histórico, pero sí hacerlo en relación a las diferentes disciplinas involucradas. Precisamente porque se considera como variables de análisis “(...) los elementos económicos, las relaciones espaciales y cierto tipo de interacciones sociales" (Van Young, 1991: 261), esto lleva a recuperar e interactuar con variadas disciplinas. Otro de los rasgos del análisis regional es que contribuye a resolver la tensión entre lo general y lo particular, brindando la posibilidad de "(...) reconciliar la microperspectiva con la macroperspectiva" (Van Young, 1991: 260). En efecto, la participación de "(...) actores sociales locales y extraregionales" (Bandieri, 1995: 282) implica dar cuenta de la diversidad, heterogeneidad y variabilidad dentro de lo que se define en el proceso de estudio mismo, como una "región".

Otra característica de los enfoques histórico-regionales, es el carácter vinculado predominantemente con las tareas de intervención (Méndez y Tozzini, 2011), atendiendo a la resolución de problemáticas concretas por parte de profesionales de diferentes disciplinas. Si bien nuestro trabajo central es de investigación, claramente ha sido en las tareas de intervención donde las herramientas del abordaje histórico-regional han resultado sumamente provechosas para complementar y profundizar lo recabado a partir del trabajo etnográfico.

Finalmente, y en directa relación con lo presentado en estos párrafos, deseamos señalar la favorable recepción de estas lecturas históricas en la región de Norpatagonia desde diferentes organizaciones sociales, políticas, sindicales, etc., precisamente por la ruptura con las conceptualizaciones tradicionales, promovidas desde los sectores de poder que difundieron las Historias nacionales y provinciales de fuerte matriz institucional. La participación de profesionales que recuperan estas perspectivas con los sectores sociales involucrados, constituye una muestra elocuente de lo dicho en estos párrafos ${ }^{5}$.

\section{Los abordajes histórico-regionales y la problemática de los movimientos indí- genas del pueblo Mapuche}

En este último apartado, consideramos la recuperación de diversas categorías y lecturas de los procesos regionales para el abordaje de los movimientos indígenas del pueblo Mapuche. Desde nuestra perspectiva, el uso de una metodología de tipo etnográfica -característica de la Antropología Social- en estrecha relación con el abordaje histórico del enfoque regional, es clave para poder interpretar procesualmente dinámicas que han ido surgiendo como relevantes a partir del trabajo etnográfico.

Además, el análisis de los procesos históricos que intervienen en los casos particulares -es decir, las dinámicas globales en las poblaciones específicas- posibilita, en nuestro caso, dar cuenta de las grandes heterogeneidades que podemos encontrar bajo la categoría de "pueblos

\footnotetext{
5 Es el caso del libro Historia de las familias Mapuche Paichil Antriao y Quintriqueo de la Costa Norte del lago Nahuel Huapi, compilado por G. Ghioldi (2009). De este trabajo han participado las propias comunidades indígenas a la que refiere la obra, la biblioteca que compiló el trabajo e investigadores de la Facultad de Filosofía y Letras de la Universidad de Buenos Aires, de la Universidad Nacional del Comahue, de la Universidad Nacional de Río Negro y un abogado especialista en la temática indígena (patrocinante legal de estas agrupaciones indígenas).
} 
indígenas" o en un mismo pueblo como el "Mapuche". De esta forma, evitamos caer en un doble reduccionismo; el de los teóricos "macro" "(...) que a menudo ignoran la diversidad etnográfica que se encuentra debajo de lo que es definido como "pueblos indígenas" y el de los "estudios de comunidad" como ejemplo para toda la región, que generalizan más allá de los límites de la unidad estudiada, ignorando "(...) el mosaico de diversas condiciones culturales, económicas e históricas que tienen un impacto diferente en cada uno de los estudios comunitarios" (Nash, 2006: 76).

En relación a nuestra problemática -centrada en dar cuenta de la emergencia, desarrollo y consolidación en los últimos años de los movimientos indígenas del pueblo Mapuche- la contextualización histórica permite considerar diferentes aspectos nodales. Uno de ellos, es la presencia desde hace décadas de diversas agrupaciones que son clave en los procesos organizativos indígenas. Es el caso de la Confederación Indígena Neuquina, conformada en los años setenta, una de las primeras agrupaciones del país. Otros casos son las organizaciones surgidas en los años ochenta con el retorno de la democracia, como "Nehuén Mapu" ("La fuerza de la tierra") de la ciudad de Neuquén, el "Consejo Asesor Indígena" de la Provincia de Río Negro o los Centros Mapuche de diversas localidades de la misma provincia. Los referentes indígenas que conformaron estos grupos, contaban con experiencias en la actividad sindical, en la militancia barrial, en partidos políticos, así como en tareas comunitarias vinculadas al obispado local (Radovich, 1992).

En el desarrollo de estas organizaciones fue central la presencia de los grupos de base de la iglesia católica que realizaban tareas de promoción social en las comunidades Mapuche y de diferentes movimientos sociales. A su vez, debemos considerar ciertas dinámicas socioeconómicas regionales: un destacado grado de desarrollo industrial y una importante presencia de empleados estatales concentrados en pocas ramas económicas (Iñigo Carrera, et al., 1999) y agrupados sindicatos poderosos (en algunos casos combativos). También destacamos la presencia de población migrante de otras regiones de Argentina y también de Chile. En este sentido, es importante señalar que esta región Norte de la Patagonia ha sido una zona de "exilio interno" durante la última dictadura militar, de militantes sociales provenientes de diferentes lugares de la Argentina y del vecino país de Chile. Las causas radican en la relativa situación de aislamiento que primaba en aquel entonces, respecto de otros centros urbanos más poblados (Buenos Aires, Rosario, Córdoba, etc.) con fuerte desarrollo industrial y/o estudiantil, donde la represión fue muy intensa. Fue clave, en este sentido, el "paraguas" protector que ofrecía a los militantes la iglesia local, representada por la emblemática figura del Monseñor Jaime De Nevares ${ }^{7}$ (Favaro y Scuri, 2005 y Aiziczon, 2005).

Esto contribuye a explicar la presencia de variadas organizaciones sociales, políticas y sindicales que surgen con el retorno de la democracia -que se consolidan en los años subsiguientes-, y son fundamentales en el apoyo a las demandas de los pueblos indígenas (al igual que otros grupos sociales).

Otro aspecto relevante en estas experiencias organizativas, son los procesos migratorios rural-urbanos de pobladores indígenas que se vienen registrando desde hace décadas, lo que resulta una dinámica nodal en la emergencia de los movimientos indígenas en las últimas tres décadas. Como ha señalado Radovich (1992), en contraste con el proceso de desadscripción étnica experimentado por sus ancestros en las décadas anteriores, los hijos y nietos de migrantes indígenas -que se fueron asentando en las diferentes urbes de la región, producto precisamente de diversas transformaciones socioeconómicas ${ }^{8}$ iniciaron un proceso de

\footnotetext{
6 El nombre de "Consejo Asesor" se debe a que estuvo integrado originalmente por promotores y técnicos que asesoraban al gobierno provincial (Radovich, 1992).

${ }^{7}$ Prueba de esta particular impronta, es que Jaime De Nevares fue el creador en la Provincia de Neuquén, de la primera delegación de la organización "APDH" (Asamblea Permanente por los Derechos Humanos) que se constituía fuera de la Ciudad de Buenos Aires (Aiziczon, 2005: 181).

${ }^{8}$ Las diversas ciudades de Norpatagonia han recibido, históricamente, importantes contingentes de población mapuche del medio rural que se fue instalando en los centros urbanos (Radovich, Balazote, 1992: 163-164). Entre las causas de estos procesos migratorios, se encuentran la imposibilidad, por parte del sector doméstico, de acrecentar las actividades
} 
revalorización de la identidad Mapuche. Nacidos y criados en el medio urbano, o llegados a corta edad a la ciudad, lograron un mejor nivel de instrucción formal y de capacitación laboral que sus mayores, pasando además por experiencias políticas como las que antes señalamos (en sindicatos, organizaciones sociales, comunidades de base de la iglesia católica, etc.) (Radovich, 1992). En plena coincidencia con lo registrado para un sinfín de casos para América Latina (Bartolomé, 1997; Bengoa, 2007), esta nueva capa de dirigentes indígenas generó una profunda transformación en las formas de relacionarse con el Estado, las agencias de desarrollo y la sociedad en su conjunto.

Por último, otro aspecto fundamental de este enfoque para abordar la problemática del pueblo Mapuche, lo constituyen las heterogeneidades regionales, considerando las “(...) temporalidades múltiples y singulares (...), que a la vez requiere ser referenciado con otras territorialidades y espacialidades para adquirir plena significación" (Méndez y Tozzini, 2011:166). Así encontramos un gran contraste entre zonas donde las comunidades Mapuche fueron tempranamente reconocidas ${ }^{9}$ y aquellas zonas -como las que son jurisdicción de Parques Nacionales ${ }^{10}$ - donde -históricamente- fue intensa la invisibilización y negación de la población indígena (García y Valverde, 2007).

\section{A modo de cierre}

A partir del recorrido efectuado en estas páginas, observamos cómo la consolidación en las últimas décadas de diferentes equipos de investigación en la región de Norpatagonia fundamentalmente del campo de la disciplina histórica-, con perspectivas alternativas y superadoras de los enfoques tradicionales, viene generando promisorios resultados. Uno de ellos, es la creciente convergencia entre diferentes disciplinas, a partir de una flexibilización de las "fronteras" que las separaban. Otro, es la revisión de las omisiones y negaciones que cada ciencia -fuertemente condicionada por la tradición positivista- había efectuado.

Las particulares condiciones de producción de conocimiento que se dieron en la disciplina histórica, de esta región -con el retorno de la democracia- posibilitaron el desarrollo y consolidación de los denominados "enfoques regionales". Así, se pusieron en discusión muchos de los preceptos, por un lado de la historiografía nacional -que ocultaba los procesos del interior del país- y por otro de las lecturas tradicionales para esta zona. Entre estos factores, se encuentra la presencia de un destacado centro académico -como es la Universidad Nacional del Comahuecon la indudable calidad de los profesionales involucrados en esta tarea, así como la articulación de los mismos (por relaciones familiares, sociales, político-ideológicas, etc.) con los procesos sociales locales y regionales. Esto explica, que cada vez más problemáticas presentes en la sociedad encontraran su correlato en innovadoras investigaciones.

Desde la Antropología, la búsqueda de lecturas más dinámicas y dialécticas, es una constante desde el retorno de la democracia. Pero a diferencia de lo sucedido con la carrera de Historia, su dictado en la Patagonia es muy reciente y la mayor parte de los trabajos son efectuados en centros académicos de otras zonas (principalmente Buenos Aires), lo que explica

productivas en la misma proporción que el crecimiento poblacional y a la vez -como sucede en todo el resto de Argentina y América Latina- en la creciente destrucción de unidades de producción familiar, en detrimento de grandes emprendimientos.

9 En década de 1960, durante la primera gestión del gobernador Felipe Sapag (destacado caudillo provincial desde aquellos años), el Estado Provincial llevó adelante la delimitación de diversas "reservas indígenas" (como se las denominaba en aquel entonces) en territorios ocupados por familias mapuche. Al finalizar la década de 1980 ascendían a más de 30 las agrupaciones formalizadas en la provincia (Falaschi, 1994: 12-13).

10 Esta región cuenta con dos vastos Parques Nacionales, que además son los primeros en fundarse en el país, el Parque Nacional Nahuel Huapi (creado en el año 1934) y el Parque Nacional Lanin (1937). El primero cuenta con una superficie de 717.261 hectáreas al Oeste de las provincias de Neuquén y Río Negro (Parque Nacional Nahuel Huapi, 2012). El segundo, cuenta con un área de 412.000 hectáreas, al oeste de la Provincia de Neuquén (Parque Nacional Lanín, 2012). 
que la influencia se dé -fundamentalmente- de la carrera de Historia, ya consolidada, hacia las restantes.

En esta línea de análisis, enfatizamos la necesidad de romper con la ahistoricidad de ciertas concepciones de "sociedad y cultura" como autoreguladas y definidas en sí mismas, como tan bien argumentara Wolf (1982), en su magnífica obra "Europa y la Gente sin Historia". Los pueblos indígenas, no son ajenos a estas características, donde -al decir de Bartolomé- "(...) no son un testimonio del pasado, un anacronismo o una 'anormalidad histórica"” (2004: 71).

Resaltamos el carácter dinámico que poseen las prácticas y relaciones sociales y a la vez, el carácter contradictorio de los procesos sociales (Achilli, 2005: 17). De allí, que "(...) adquiere relevancia reconocer en los sujetos sus prácticas, sus experiencias, los modos de constitución de los distintos espacios, distintas relaciones, distintas conflictividades" (2005: 17). Ahora bien, continuando con este eje argumentativo, “(...) las prácticas y representaciones que generan los sujetos son heterogéneas" (Achilli, 2005: 17). Esto es explicable en la medida que en ellas se dan "(...) experiencias sociales e históricas diferenciadas, huellas del pasado, intentos de transformarlas y construcciones de sentidos en relación a lo vivido y al porvenir" (Achilli, 2005: 25). Es necesario por lo tanto, que dicha heterogeneidad se la "inscriba históricamente", en la medida en que las condiciones pasadas constituyen "(...) un horizonte de posibilidades latentes, una jaula flexible e invisible para ejercer dentro de ella la propia libertad condicionada" (Achilli, 2005: 25), tal como sostiene esta autora parafraseando a C. Ginzburg en su célebre obra El queso y los gusanos (1999, original de 1976).

Demás está aclarar, en este punto de nuestra argumentación, la trascendencia de procesos -como las migraciones rural-urbanas y la presencia regional de diversas organizaciones solidarias con los pueblos indígenas- en la conformación, como sujetos sociales, del pueblo Mapuche a través de sus movimientos. En otras palabras, vuelve a cobrar pleno sentido la afirmación de Sidney Mintz, acerca del poder explicativo de la Antropología, que -sin la Historia- se ve gravemente comprometido. Como tan lúcidamente planteara ese autor en su trabajo Dulzura y poder. El lugar del azúcar en el mundo moderno -cuya cita elegimos como epígrafe de este artículo- "(...) sin la Historia el poder explicativo [de la Antropología] se ve gravemente comprometido" (1996: 28), sin por ello aceptar "(...) acríticamente el mandato de que la Antropología debe convertirse en Historia o no ser nada".

Pero recuperar tal complejidad de lo social, supone transitar distintas dificultades, además de los riesgos de reproducir algunas polarizaciones que dualizan y reducen el análisis (Achilli, 2005: 26), como las que también señalara Nash (2006) en relación a los pueblos indígenas en México. Es el caso de las dicotomizaciones "entre un sujeto pasivo" (en la medida en que sólo se ve la estructura) o "(...) un sujeto que voluntariamente es hacedor de lo social" (soslayando la estructura) (Achilli 2005: 26).

En este recorrido que venimos efectuando hace años desde la Antropología, encontramos en la perspectiva histórico-regional, una fuente teórico-metodológica sumamente propicia para "transitar" lo más acertadamente posible las dificultades que se presentan. La no fragmentación a priori de la unidad de estudio espacio-temporal a abordar, la mirada interdisciplinaria, la articulación macro y micro, la consideración de los pueblos indígenas como preocupación de la Historia y el trabajo de investigación -fuertemente orientado a la intervención- son aristas en las que se hace particularmente proclive esta articulación. En relación a este último aspecto, deseamos señalar, tal como lo planteara Miguel Bartolomé, el rol que desempeña la etnografía en las "sociedades plurales", donde hasta la misma "investigación pura" y sin un aparente "fin inmediato" ha acompañado y contribuido a todas las grandes transformaciones (2004: 71).

Por ello, entendemos que parte de este recorrido debe ayudar a construir una "nueva Historia" que incluya al pueblo Mapuche como parte fundamental "del relato histórico de la Patagonia" y que contribuya a recuperar su existencia en el pasado, en el presente y como parte de los proyectos a futuro. 


\section{Agradecimientos}

Este trabajo se enmarca en el proyecto UBACyT 2010-2012: "Conflictividad y trayectorias organizativas de indígenas y pequeños productores criollos en los últimos remanentes de tierras forestales: una perspectiva comparativa entre el norte y sur de la Argentina”, Facultad de Filosofía y Letras, Universidad de Buenos Aires. Agradecemos a la Lic. Zuleika Crosa por sus valiosos comentarios. 


\section{REFERENCIAS}

Achilli, E. (2005). Investigar en Antropología Social. Los desafios de transmitir un oficio. Rosario: Laborde Libros Editor.

Aiziczon, F. (2005). "Neuquén como campo de protesta”. En: O. Favaro (Ed.), Sujetos Sociales y Política. Historia Reciente de la Norpatagonia Argentina (pp. 175-201). Buenos Aires: Editorial La Colmena.

Assadourian, C. S. (1982). Mercado interno, regiones y espacio económico. Lima: Instituto de Estudios Peruanos.

Bartolomé, M. A. (2004). "Movilizaciones étnicas y crítica civilizatoria. Un cuestionamiento a los proyectos estatales en América Latina". Perfiles Latinoamericanos, 24, 85-105.

- (1997). Gente de costumbre y gente de razón: las identidades étnicas en México. México: Siglo XXI.

Bandieri, S. (2009). "Cuando crear una identidad nacional en los territorios patagónicos fue prioritario". Revista Pilquen. Sección Ciencias. Sociales 11, 1-5.

- (2005). Historia de la Patagonia. Buenos Aires: Editorial Sudamericana.

- (2001a). "Haciendo Historia regional. Pueblos y Fronteras de la Patagonia Andina". Revista de Ciencias Sociales 2, 4-6.

- (Coord.) (2001b). Cruzando la cordillera. La frontera argentino-chilena como espacio social. Neuquén: CEHIR, Universidad Nacional del Comahue.

- (1995). "Acerca del concepto de región y la historia regional: la especificidad de la Norpatagonia". Revista de Historia 5, 277-293.

- (1993a). "Condicionantes Históricos del Asentamiento Humano después de la ocupación militar del espacio". En: S. Bandieri, O. Favaro, M. Morinelli (Comps.), Historia de Neuquén (pp. 109-146). Buenos Aires: Plus Ultra.

- (1993b). "Actividades económicas y modalidades de asentamiento". En: S. Bandieri, O. Favaro, M. Morinelli (Comps.), Historia de Neuquén (pp. 109-146). Buenos Aires: Plus Ultra.

Bandieri, S., Blanco, G. y Varela, G. (2006). Hecho en Patagonia. La Historia en perspectiva regional. Neuquén: Ediciones CEHIR-UNCo.

Bandieri, S., Favaro, O. y Morinelli, M. (Comps.). (1993). Historia de Neuquén. Buenos Aires: Plus Ultra.

Bengoa, J. (2007). La Emergencia Indígena en América Latina. Santiago de Chile: Fondo De Cultura Económica.

Carbonari, M.R. (2009). "De cómo explicar la región sin perderse en el intento. Repasando y repensando la Historia Regional”. História Unisinos 13(1), 19-34.

Cardoso, C.F.S. (1984). Economía e sociedade em áreas coloniais periféricas: Guiana Francesa e Pará: 1750-1817. Río de Janeiro: Graal.

Chiaramonte, J. C. (1983). Formas de sociedad y economía en Hispanoamérica. México: Grijalbo.

Colectivo Editor Revista de Historia (2011). "Sobre la profesión y sus proyectos editoriales. A propósito del trigésimo aniversario de la publicación del Departamento de Historia". Revista de Historia 12, 1-13.

De Jong, G. (2009). Geografía, Método Regional y Planificación. Buenos Aires: Editorial Catálogos.

Donghi, T. H. (1972). Revolución y guerra. Buenos Aires: Siglo XXI.

Falaschi, C. (1994). La Confederación Indígena Neuquina. Neuquén: Serie La tierra Indígena Americana. IREPS-APDH.

Favaro, O. (2005). Sujetos Sociales y Politica. Historia Reciente de la Norpatagonia Argentina. Buenos Aires: Editorial La Colmena. 
Favaro, O., Scuri, M. C. (2005). "La trastienda de la Historia Regional". En: O. Favaro (Ed.), Sujetos Sociales y Política. Historia Reciente de la Norpatagonia Argentina (pp. 11-20). Buenos Aires: Editorial La Colmena.

Garavaglia, J. C. (1983). Mercado Interno y economía colonial. México: Grijalbo.

García, A., Valverde, S. (2007). "Políticas estatales y procesos de etnogénesis en el caso de poblaciones mapuche de Villa La Angostura-Neuquén-Argentina". Cuadernos de Antropología Social. 25, 111-132.

Ghioldi, G. (2009). Historia de las familias Paichil Antriao y Quintriqueo de la Costa Norte del lago Nahuel Huapi. Neuquén: Archivos del Sur - Biblioteca Popular Osvaldo Bayer.

Ginzburg, C. (1999). El queso y los gusanos. El cosmos, según un molinero del siglo XVI. Barcelona: Muchnik Editores S.A.

INDEC - Instituto Nacional de Estadística y Censos (2004-2005). Encuesta Complementaria de Pueblos Indigenas (ECPI). Argentina.

Juliano, D. (1996). "Los mapuches, la más larga resistencia”. Anuario del IEHS 11, 303-327.

Iñigo Carrera, I., Podestá, J. y Cotarelo, M. C. (1999). "Las estructuras económico sociales concretas que constituyen la formación económica de la Argentina". PIMSA, Publicación del Programa de Investigación sobre el Movimiento de la Sociedad Argentina.

Mandrini, R. (2007). "La historiografía argentina, los pueblos originarios y la incomodidad de los Historiadores". Quinto Sol 11.

Méndez, L. (2010). Estado, frontera y turismo. Buenos Aires: Prometeo Libros.

- (2009). "El Estigma de origen. Chilenos e indígenas en el gran lago 1880-1935". En: G. Ghioldi, (Comp.) Historia de las familias Paichil Antriao y Quintriqueo de la Costa Norte del lago Nahuel Huapi. Neuquén: Archivos del Sur - Biblioteca Popular Osvaldo Bayer.

- (2006). "Circuitos económicos en el Gran Lago. La región del Nahuel Huapi entre 1880 y 1930”. En: S. Bandieri, G. Blanco, G. Varela (Eds.), Hecho en Patagonia. La Historia en perspectiva regional. Neuquén: Ediciones CEHIR-UNCo.

- (2005). "Una región y dos ciudades. Puerto Montt y Bariloche: una Historia económica compartida”. Pueblos y Fronteras de la Patagonia andina 6, 4-11.

Méndez, L. y Tozzini, A. (2011). "De espacialidades y temporalidades en la Norpatagonia Andina. Algunos aportes para su construcción y estudio". En: P. Navarro Floria, W. Delrio (Eds.), Cultura y Espacio. Araucanía y Norpatagonia (pp. 158-171). San Carlos de Bariloche: Universidad Nacional de Río Negro. Instituto de investigaciones en Diversidad Cultural y Procesos de Cambio.

Mintz, S. (1996). Dulzura y poder. El lugar del azúcar en el mundo moderno. México: Siglo XXI.

Nash, J. (2006). Visiones Mayas: el problema de la autonomía en la era de la globalización. Buenos Aires: Antropofagia.

Navarro Floria, P. (2008). "El proceso de construcción social de la región del Nahuel Huapi en la práctica simbólica y material de Exequiel Bustillo (1934-1944)”. Revista Pilquen, Sección Ciencias Sociales 10.

- (1999). Historia de la Patagonia. Buenos Aires: Editorial Ciudad.

Parque Nacional Lanin. (2012, Marzo 23). Disponible: http://www.parquenacional lanin.gov.ar/

Parque Nacional Nahuel Huapi. (2012, Marzo 23). Disponible: http://www.nahuelhuapi.gov.ar/

Radovich, J. C. (2003). Impacto Social de grandes aprovechamientos hidroenergéticos sobre comunidades rurales de norpatagonia. Tesis de Doctorado, Universidad de Buenos Aires, Facultad de Filosofía y Letras, Argentina.

- (1992). "Política Indígena y Movimientos Étnicos: el caso Mapuche". Cuadernos de Antropología 4, 47-65. 
Radovich, J. C. y Balazote, A. (2009). "El pueblo mapuche contra la discriminación y el etnocidio". En: G. Ghioldi (Comp.), Historia de las familias Paichil Antriao y Quintriqueo de la Costa Norte del lago Nahuel Huapi (pp. 35-59). Neuquén: Archivos del Sur - Biblioteca Popular Osvaldo Bayer.

- (1992). "El pueblo Mapuche hoy". En: J. C. Radovich, A. Balazote (Eds.), La problemática indígena (pp. 159-186). Buenos Aires: CEDAL.

Rockwell, E. (2009). La Experiencia Etnográfica. Historia y cultura en los procesos educativos. Buenos Aires: Editorial Paidós.

Van Young, E. (1991). "Haciendo Historia regional: Consideraciones metodológicas y teóricas". En: P. Pérez Herrero (Comp.), Región e Historia en México (1700-1850) (pp. 99-122). México: Instituto Mora-UAM.

Viazzo, P. P. (2003). Introducción a la antropología histórica. Lima: Pontificia Universidad Católica del Perú, Instituto Italiano de Cultura.

Wolf, E. (1982). Europa y la gente sin historia. Buenos Aires: Fondo de Cultura Económica de Argentina.

\section{SOBRE EL AUTOR}

Sebastián Valverde: es Doctor (2006) de la Universidad de Buenos Aires, orientación en Antropología (año 2006) de Filosofía y Letras (UBA). Es Licenciado y Profesor en Ciencias Antropológicas de la misma Facultad. Es Investigador, con categoría Asistente del Consejo Nacional de Investigaciones Científicas y Técnicas (CONICET) con sede de trabajo en el Instituto de Ciencias Antropológicas de la mencionada Facultad. También es docente del Departamento de Ciencias Antropológicas y de la Maestría en Antropología Social (FFyL-UBA). Sus temas de investigación se basan en la antropología económica, relaciones interétnicas y pueblos indígenas, en particular relacionados al pueblo indígena Mapuche asentado en el sur argentino. Ha publicado sobre estos temas en artículos en revistas con referato, Capítulos de libros y Libros nacionales e internacionales, además de diversas presentaciones en eventos científicos nacionales e internacionales. Ha coordinado 3 proyectos de investigación en relación a estos temas de la Agencia Nacional de Promoción Científica y de la Universidad de Buenos Aires y ha integrado en otros proyectos de diversos organismos. Ha coordinado e integrado diferentes proyectos de transferencia y extensión, financiados por la UBA, el Ministerio de Educación de la Nación y el Instituto Nacional de Asuntos Indígenas (INAI). 